\title{
A qualidade dos carboidratos dietéticos está associada à adequação do consumo de minerais: um estudo com mulheres acompanhadas no SUS
}

\author{
The quality of dietary carbohydrates is associated with the adequacy of mineral consumption: \\ a study with women followed at SUS
}

\section{DOI: $10.37111 /$ braspenj.2019344001}

Leandro Teixeira Cacau

Ayana Florêncio de Meneses ${ }^{2}$

Helena Alves de Carvalho Sampaio ${ }^{3}$

Antônio Augusto Ferreira Carioca ${ }^{4}$

Ana Luiza de Rezende Ferreira Mendes

Luiz Gonzaga Porto Pinheiro ${ }^{6}$

Daianne Cristina Rocha

llana Nogueira Bezerra ${ }^{8}$

\section{Unitermos:}

Consumo de alimentos. Minerais. Carboidratos. Mulheres.

\section{Keywords:}

Food consumption. Minerals. Carbohydrates. Women.

\section{Endereço para correspondência:}

Leandro Teixeira Cacau

Rua Maestro Carlos Cruz, 116 - Vila Indiana - São

Paulo, SP, Brasil - CEP 05585-020

E-mail: Icacau@usp.br

\section{Submissão}

8 de junho de 2019

Aceito para publicação

14 de setembro de 2019

\begin{abstract}
RESUMO
Introdução: As inadequações na ingestão de micronutrientes são um problema emergente em termos de saúde pública. Recentemente, o papel dos macronutrientes foi proposto como fator relacionado à adequação do consumo de vitaminas e minerais. $\mathrm{O}$ objetivo deste estudo foi avaliar se a qualidade dos carboidratos da dieta está associada com o consumo adequado de minerais. Método: Estudo transversal realizado com mulheres acompanhadas em um serviço de mastologia do SUS, situado na capital do estado do Ceará. A coleta de dados foi realizada entre os anos de 2016 e 2017. Neste trabalho, foram analisados os dados de consumo alimentar investigados por meio da aplicação em duplicata do recordatório de 24 horas, sendo um deles referente a um dia de final de semana. Os dados obtidos foram digitados na Plataforma Brasil Nutri e lançados no Statistical Analysis System. Os dados foram corrigidos para variância intrapessoal no programa estatístico Multiple Source Method para predição da ingestão habitual. Foram avaliados o consumo de zinco $(\mathrm{Zn})$, selênio $(\mathrm{Se})$, cobre $(\mathrm{Cu})$, manganês $(\mathrm{Mn})$, magnésio $(\mathrm{Mg})$, ferro $(\mathrm{Fe})$ e fósforo $(\mathrm{P})$. Para avaliar a qualidade dos carboidratos, calculou-se o Índice de Qualidade do Carboidrato (IQC). As análises estatísticas foram realizadas no SPPS 20.0 e considerou-se significante valores de $p<0,05$. $O$ projeto foi aprovado pelo Comitê de Ética em Pesquisas da instituição de origem. Resultados: No total, 648 mulheres foram avaliadas, com idade média de 52,4 $\pm 9,3$ anos. A média do IQC foi de $12 \pm 3,1$. Maiores pontuações do IQC foram associadas com maior adequação de consumo de $\mathrm{Zn}(\mathrm{p}<0,001), \mathrm{Mn}(\mathrm{p}<0,001), \mathrm{Mg}(\mathrm{p}<0,001), \mathrm{Cu}(\mathrm{p}<0,001), \mathrm{P}(\mathrm{p}=0,002)$ e de Fe $(p=0,006)$, mas não para $\mathrm{Se}(p=0,711)$. Conclusões: Os achados deste estudo demonstraram que maiores valores de IQC estão associados com maior adequação do consumo dos minerais avaliados, com exceção do selênio.
\end{abstract}

\section{ABSTRACT}

Introduction: Inadequate micronutrient intake is an emerging public health problem. Recently the role of macronutrients has been proposed as a related factor in the adequacy of vitamin and mineral intake. The aim of this study was to evaluate the association between dietary carbohydrate quality and adequate mineral intake. Methods: Cross-sectional study conducted with women followed at a SUS mastology service located in the state capital of Ceará. Data collection was performed between 2016 and 2017. In this studyw we analyzed the food consumption data investigated through the duplicate application of the 24-hour recall, one of them referring to a weekend day. The data obtained were entered in the Brazil Nutri Platform and released in the Statistical Analysis System. Data were corrected for intrapersonal variance in the Multiple Source Method statistical program to predict habitual intake. The consumption of zinc ( $\mathrm{Zn})$, selenium (Se), copper (Cu), manganese $(\mathrm{Mn})$, magnesium $(\mathrm{Mg})$, iron $(\mathrm{Fe})$ and phosphorus $(\mathrm{P})$ were evaluated. To assess carbohydrate quality, the Carbohydrate Quality Index (CQI) was calculated. Statistical analyzes were performed using SPPS 20.0 and $p<0.05$ was considered significant. The project was approved by the Research Ethics Committee of the institution. Results: In total, 648 women were evaluated, with a mean age of $52.4 \pm 9.3$ years. The average IQC was $12 \pm 3.1$. Higher CQI scores were associated with higher intake adequacy of $\mathrm{Zn}(\mathrm{p}<0.001), \mathrm{Mn}(\mathrm{p}<0.001), \mathrm{Mg}$ (p $<0.001), \mathrm{Cu}(\mathrm{p}<0.001), \mathrm{P}(\mathrm{p}=0.002)$ and $\mathrm{Fe}(\mathrm{p}=0.006)$, but not for $\mathrm{Se}(\mathrm{p}=0.711)$. Conclusions: The findings of this study showed that higher $C Q I$ values are associated with greater adequacy of minerals intake, except selenium.

1. Doutorando em Nutrição em Saúde Pública, Faculdade de Saúde Pública, Universidade de São Paulo, São Paulo, SP, Brasil.

2. Doutoranda em Saúde Coletiva, Departamento de Nutrição, Universidade Estadual do Ceará (UECE), Fortaleza, CE, Brasil.

3. Doutora em Farmacologia, Professora Emérita da UECE. Professora permanente do Programa de Pós-Graduação em Saúde Coletiva, UECE, Fortaleza, CE, Brasil.

4. Doutor em Nutrição em Saúde Pública, Departamento de Nutrição, Universidade de Fortaleza, Fortaleza, CE, Brasil.

5. Doutora em Saúde Coletiva, Departamento de Nutrição, Centro Universitário Estácio do Ceará, Fortaleza, CE, Brasil.

6. Doutor em Cirurgia, Faculdade de Medicina, Universidade Federal do Ceará, Fortaleza, CE, Brasil.

7. Doutora em Saúde Coletiva, Departamento de Nutrição, Centro Universitário UniChristus, Fortaleza, CE, Brasil.

8. Doutora em Fisiopatologia Clínica e Experimental, Departamento de Nutrição, UECE, Fortaleza, CE, Brasil. 


\section{INTRODUÇÃO}

Ao longo dos últimos anos, os estudos vêm ressaltando a importância da avaliação da qualidade da dieta das populações de uma maneira holística, pautados no conceito de que a dieta deve ser considerada de maneira integrada, ao invés de avaliações isoladas de determinados nutrientes'.

As inadequações na ingestão de micronutrientes são um problema emergente em termos de saúde pública. No Brasil, os principais micronutrientes com elevadas inadequações no consumo são as vitaminas $A, E$ e $C$ e os minerais magnésio e zinco, na população adulta e idosa ${ }^{2-4}$.

Intervenções que assegurem a adequação do consumo dos micronutrientes são de interesse, tendo em vista que estes nutrientes participam de diversas funções no organismo, com participação no sistema antioxidante e imunológico, dentre outras funções, além de estarem relacionados com doenças crônicas não-transmissíveis e doenças carenciais 5 .

O papel dos macronutrientes vem sendo estudado como um possível fator relacionado à adequação do consumo de micronutrientes, sobretudo o papel desempenhado pelos carboidratos. Estudos prévios investigaram a associação da quantidade de carboidratos com a adequação do consumo de micronutrientes, mas são escassas as evidências da associação com a qualidade dos carboidratos da dieta com a adequação do consumo de micronutrientes, em específico com os minerais ${ }^{6-9}$.

Diversos componentes de avaliação da qualidade dos carboidratos foram propostos, como o índice glicêmico, a carga glicêmica, o consumo de fibras, de grãos integrais e de açúcares de adição ${ }^{10}$. Deste modo, foi o proposto o Índice de Qualidade do Carboidratos (IQC), como meio de avaliar a qualidade dos carboidratos da dieta utilizando como métricas o índice glicêmico, a ingestão de fibras, a relação de grãos integrais por grãos totais e a relação de carboidrato sólido por carboidratos totais ${ }^{11}$.

Em nosso conhecimento, estudos que avaliem a adequação do consumo de micronutrientes com a qualidade dos carboidratos aferida com base no IQC são escassos. Deste modo, o objetivo deste trabalho é de investigar se a maior qualidade dos carboidratos está relacionada com a adequação do consumo de minerais da dieta de mulheres acompanhadas por um serviço do Sistema Único de Saúde (SUS).

\section{MÉTODO}

Trata-se de um estudo com delineamento transversal que utiliza dados provenientes do Projeto Prevendo: Saúde, EnveIhecimento, Dieta e Inflamação: desenvolvimento, validação e normatização de instrumentos para promoção da saúde e prevenção de doenças crônicas não-transmissíveis, que, por sua vez, possui como principal objetivo a normatização do uso de indicadores na rotina de atendimento do SUS, objetivando um maior sucesso na promoção da saúde e no combate as doenças crônicas não-transmissíveis. $\bigcirc$ projeto supracitado foi aprovado pelo Comitê de Ética de Pesquisas com Seres Humanos da Universidade Estadual do Ceará, sob o número de protocolo 314.351 . Todas as participantes assinaram um termo de consentimento livre e esclarecido (TCLE) e foram informadas de maneira tácita sobre os objetivos da pesquisa.

A coleta de dados aconteceu entre os anos de 2016 e 2017 e foi realizada no Grupo de Educação e Estudos Oncológicos (GEEON), uma organização não-governamental filiada à Faculdade de Medicina da Universidade Federal do Ceará. O GEEON atua como um Serviço de Referência para Diagnóstico em Mastologia (SDM) do SUS.

Os critérios de inclusão no estudo foram: mulheres que consentissem em participar do estudo, com idade igual ou superior a 18 anos, que não possuíssem doença que impedisse a participação nas etapas do estudo, que estivessem em atendimento pelo SUS e que realizassem mamografias no serviço. A presença de gravidez foi considerada como critério de exclusão. Foram coletados dados de identificação, socioeconômicos, de antropometria e de consumo alimentar.

Em relação aos dados de antropometria, foram coletados peso e altura das participantes do estudo. Foi utilizada balança antropométrica marca Filizola com estadiômetro acoplado, com capacidade de $200 \mathrm{~kg}$ (intervalo de 100 g) e $2 \mathrm{~m}$ (intervalo de $1 \mathrm{~cm}$ ). $O$ peso foi mensurado com a participante descalça, ereta, com os calcanhares juntos, sem esticar ou encolher a cabeça, olhando fixamente para frente, com braços estendidos para baixo junto ao corpo. A altura foi medida a partir do estadiômetro acoplado na balança, onde a participante esteve descalça, na posição em pé com o olhar apontando para o horizonte. $O$ índice de massa corporal (IMC) [peso $(\mathrm{kg})$ / altura ao quadrado $\left(\mathrm{m}^{2}\right)$ ] foi calculado com base nas medidas de peso e altura, e as participantes foram classificadas de acordo com a Organização Mundial da Saúde ${ }^{12}$.

Foram aplicados dois recordatórios alimentares de 24 horas (R24h), como forma de levantar informações acerca do consumo alimentar das participantes do estudo. A aplicação dos R24hs foi realizada por nutricionistas e estudantes de nutrição devidamente treinados. O primeiro R24h foi conduzido no momento da entrevista, já o segundo, através de contato telefônico ou por meio de visita domiciliar. Um dos dois R24h referiu-se a um dia de final de semana. Os dados obtidos foram digitados na Plataforma Brasil Nutri e, posteriormente, lançados no Statistical Analysis System 
(SAS). A tabela de composição nutricional utilizada foi a Tabela de Composição Nutricional dos Alimentos Consumidos no Brasil, elaborada pela Pesquisa de Orçamentos Familiares (POF) de 2008-2009'13.

Como meio de corrigir a variância intrapessoal, os dados de composição nutricional foram lançados no programa estatístico Multiple Source Method (MSM) ${ }^{14}$ para predição da ingestão habitual. Os dados de consumo referentes aos minerais foram analisados por meio da Necessidade Média Estimada (EAR) do Instituto de Medicina (IOM), de acordo com gênero e idade ${ }^{15}$.

A qualidade dos carboidratos foi aferida através do índice de qualidade dos carboidratos (IQC) ${ }^{11}$, que utiliza quatro critérios para seu cálculo, a saber: (1) o índice glicêmico (IG), (2) a ingestão total de fibra alimentar (g/d), (3) a relação de carboidratos sólidos por carboidratos totais (sólidos + líquidos) e (4) a relação de carboidratos de grãos integrais por carboidratos de grãos totais (grãos integrais + refinados + produtos preparados com farinhas refinadas).

Os carboidratos líquidos foram estimados pelo somatório dos valores de carboidratos provenientes de bebidas açucaradas e suco de frutas, enquanto os carboidratos sólidos corresponderam ao conteúdo de carboidratos dos demais alimentos com qualquer teor de carboidratos. Cada um dos critérios do IQC recebeu uma pontuação variando de 1 a 5 e, finalmente, foram somados os valores, sendo determinado então o IQC, que varia de 4 a 20 pontos (Quadro 1).

Foram realizadas análises descritivas das variáveis, com média e desvio padrão para as variáveis quantitativas e distribuição de frequência com seus respectivos intervalos de confiança de 95\% (IC95\%) para as categorias de adequação do consumo. Para testar a hipótese de associação do IQC com a adequação de cada mineral avaliado, conduziu-se análise por meio de comparação de médias com o teste Mann-Whitney. As análises estatísticas foram realizadas no software SPSS versão 20.0 e considerou-se como significante valores de $p<0,05$.

\section{RESULTADOS}

No total, foram avaliadas 648 mulheres, com idade média de 52,4 $\pm 9,3$ anos. Destas, 49,5\% completaram menos de 8 anos de estudo, $80 \%$ declararam serem não brancas, $91,5 \%$ relataram não fumarem e $80,3 \%$ apresentavam-se acima do peso (Tabela 1).

Quando avaliada a adequação dos minerais em relação à qualidade dos carboidratos, foi observado que maiores pontuações médias no IQC estavam associadas com adequação do consumo dos minerais avaliados nas mulheres

Quadro 1 - Critérios usados na determinação do índice de qualidade do carboidrato.

\section{Componentes do índice dietético Intervalo de índice (pontos) Critérios para índice mínimo Critérios para índice máximo}

Índice de qualidade do carboidrato

\begin{tabular}{|c|c|c|}
\hline Ingestão de fibras alimentares (g/d) & $1-5$ & $\begin{array}{l}\text { Ingestão mínima de fibra alimentar } \\
\text { (primeiro quintil) }\end{array}$ \\
\hline
\end{tabular}
(primeiro quintil) alimentares (quinto quintil)

$\begin{array}{lcc}\text { Índice glicêmico } & 1-5 & \begin{array}{c}\text { Índice glicêmico máximo } \\ \text { (quinto quintil) }\end{array} \\ \text { Índice glicêmico mínimo } \\ \text { (primeiro quintil) }\end{array}$

$\begin{array}{lccc}\text { Relação de grãos integrais (grãos } & 1-5 & \begin{array}{c}\text { Valor mínimo dessa relação } \\ \text { (primeiro quintil) }\end{array} & \begin{array}{c}\text { Valor máximo dessa relação } \\ \text { integrais - grãos refinados ou seus }\end{array} \\ \text { (quinto quintil) }\end{array}$
produtos)

$\begin{array}{lcc}\text { Relação de carboidratos sólidos } & 1-5 & \begin{array}{c}\text { Valor mínimo dessa relação } \\ \text { (carboidratos sólidos }- \text { carboidratos quintil) }\end{array}\end{array}$
Valor máximo dessa relação (quinto quintil)

líquidos) 
acompanhadas pelo serviço de mastologia do SUS. $\mathrm{Na}$ Figura 1, é possível visualizar os intervalos de confiança de 95\% (IC95\%) de acordo com a média do IQC em relação às categorias de adequação de consumo. Na Tabela 2, são demonstrados os valores de média, desvio padrão e valores de p-valor da associação entre oIQC e adequação do consumo dos minerais avaliados.

Tabela 1 - Características socioeconômica das 648 mulheres acompanhadas em um serviço de mastologia do SUS, Fortaleza, 2019.

\begin{tabular}{lccc}
\hline & $\mathbf{n}$ & $\%$ & Média (DP) \\
\hline Idade (anos) & 648 & - & $52,4(9,3)$ \\
\hline $\begin{array}{l}\text { Anos de estudo } \\
\quad 8\end{array}$ & 321 & $49,5 \%$ & - \\
$9-11$ & 258 & $39,8 \%$ & - \\
$\quad>12$ & 69 & $10,7 \%$ & - \\
\hline Cor (auto-referida) & & & \\
$\quad$ Branca & 130 & $20,0 \%$ & - \\
$\quad$ Não-branca & 518 & $80,0 \%$ & - \\
\hline Tabagismo & & & \\
$\quad$ Fumante & 55 & $8,50 \%$ & - \\
$\quad$ Não fumante & 593 & $91,5 \%$ & - \\
\hline IMC & & & - \\
$\quad$ Adequado & 126 & $19,7 \%$ & - \\
$\quad$ Excesso de peso & 515 & $80,3 \%$ & - \\
\hline
\end{tabular}

$\mathrm{DP}=$ desvio padrão; Não-brancas= pardas, morenas e negras; $\mathrm{IMC}=$ índice de massa corporal $\left(\mathrm{kg} / \mathrm{m}^{2}\right), 641$ mulheres.
Tabela 2 - Associação entre o IQC e a adequação do consumo dos minerais avaliados nas dietas de 648 mulheres acompanhadas em um serviço de mastologia do SUS, Fortaleza, 2019 (Valores expressos em médias e desvio padrão).

\begin{tabular}{lccc}
\hline Adequação do consumo & \multicolumn{3}{c}{ Índice de Qualidade do Carboidrato } \\
\hline & \multicolumn{1}{c}{ Média } & DP & $\mathbf{p}^{*}$ \\
\cline { 2 - 4 } Selênio ( $\boldsymbol{\mu g})$ & 12,0 & 3,2 & 0,711 \\
Adequado & 12,8 & 3,4 & \\
Inadequado & & & $<0,001$ \\
\hline Zinco (mg) & 12,3 & 3,2 & \\
Adequado & 11,1 & 3,0 & \\
Inadequado & & & $<0,001$ \\
\hline Magnésio (mg) & 13,9 & 3,4 & \\
Adequado & 11,8 & 3,1 & \\
Inadequado & & & 0,002 \\
\hline Fosforo (mg) & 12,1 & 3,2 & \\
Adequado & 10,3 & 2,4 & \\
Inadequado & & & 0,006 \\
\hline Ferro (mg) & 12,3 & 3,2 & \\
Adequado & 11,5 & 3,0 & \\
Inadequado & & & $<0,001$ \\
\hline Cobre (mg) & 12,7 & 3,1 & \\
Adequado & 10,4 & 2,8 & \\
Inadequado & & & \\
\hline Manganês (mg) & 13,1 & 3,0 & \\
Adequado & 3,0 & \\
Inadequado & 11,1 & & \\
\hline
\end{tabular}

*Teste Mann-Whitney. Mg= miligramas, $\mu g=$ microgramas. $\mathrm{DP}=$ desvio padrão.

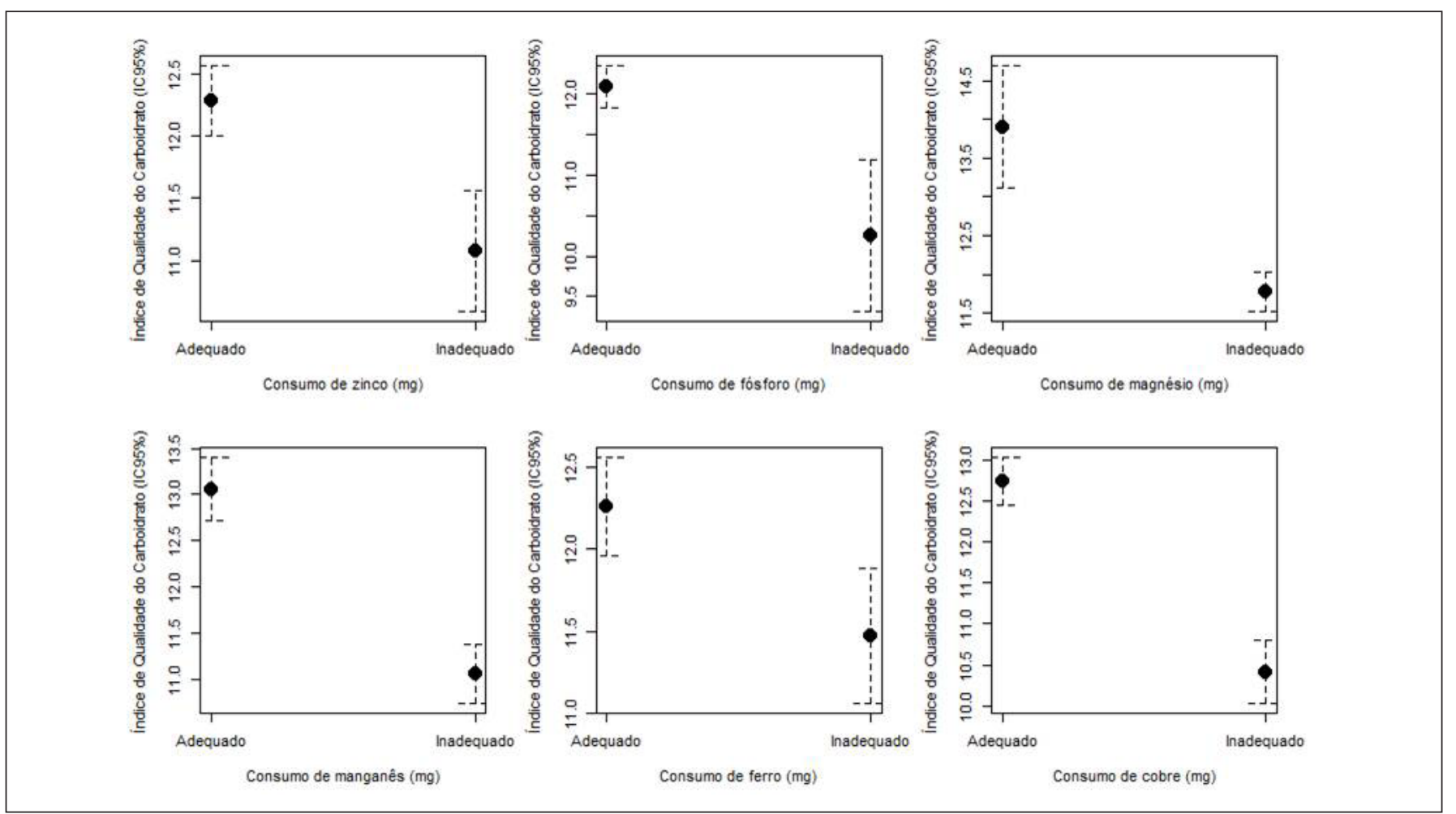

Figura 1 - Comparação de médias e seus respectivos intervalos de confiança para as categorias de adequação do consumo em relação ao IQC. Fortaleza, 2019. 
Os minerais que mais possuíram significância estatística com a qualidade dos carboidratos foram o zinco, o magnésio, o manganês e o cobre.

\section{DISCUSSÃO}

Até onde sabemos, o presente estudo é pioneiro em investigar a associação entre a qualidade dos carboidratos aferida pelo IQC com a associação de adequação de consumo de minerais da dieta de mulheres acompanhadas em serviço de referência em mastologia do SUS. Os achados deste estudo demonstraram que, quanto maior a média de pontuação no IQC, maior a adequação de consumo dos minerais avaliados nesta população.

Os carboidratos representam a maior proporção de macronutrientes da dieta e são, deste modo, bons fornecedores de micronutrientes. Os carboidratos podem ser avaliados quanto à sua qualidade, de acordo com o índice glicêmico, a carga glicêmica, a quantidade de fibras e de açúcar adicionado ${ }^{11,16}$.

Em relação ao papel dos carboidratos na adequação do consumo de micronutrientes, foram propostos intervalos de $50 \%-54 \%$ de carboidratos da energia total para uma boa adequação ${ }^{16}$.

Em um estudo realizado na Espanha, com mais de 16 mil participantes, os autores observaram que valores elevados do IQC estão mais propensos a ocasionar maior adequação de consumo dos micronutrientes ${ }^{11}$, resultado este similar ao nosso estudo. Um maior IQC está associado a menores valores de índice glicêmico, carga glicêmica e maiores em fibras, características estas inerentes a frutas, hortaliças e nozes, alimentos que são fontes de diversos minerais'. Deste modo, - IQC demonstra que a qualidade do carboidrato pode ser mais relevante do que a quantidade, onde um aumento no consumo de grãos integrais, frutas e hortaliças reduzirá efeitos adversos à saúde, aumentando a adequação dos minerais.

Estudos avaliando a adequação do consumo de minerais com a qualidade dos carboidratos são escassos. Contudo, Araújo et al. ${ }^{4}$ investigaram a inadequação de micronutrientes nos estados brasileiros e observaram que indivíduos residentes da região Sul do país possuíam maior inadequação de consumo de magnésio, enquanto na região Nordeste, os indivíduos apresentaram maior prevalência de inadequação para zinco e ferro, especialmente as mulheres. Estes autores também investigaram o consumo de macronutrientes e como resultado obtiveram que o percentual médio energético oriundo dos carboidratos foi de $54,4 \%$ para o território nacional, e especificamente na região Nordeste, o percentual médio foi de $56,1 \%$.

O IQC já foi avaliado em diferentes populações e situações, como no risco de depressão ${ }^{17}$, de obesidade ${ }^{18}$, de hipertensão ${ }^{18}$ e de doenças cardiovasculares ${ }^{19}$. Os resultados destes estudos supracitados demonstraram que uma má qualidade de carboidratos está associada com maior risco de depressão e que maiores pontuações do IQC estão associadas com menor risco de obesidade, hipertensão e de doenças cardiovasculares.

Em suma, neste estudo, uma maior média no IQC esteve associada com maior adequação no consumo de zinco, magnésio, fósforo, ferro, cobre, selênio e manganês. Contudo, estes achados devem ser vistos à luz de algumas limitações. A saber, os dados apresentados são oriundos de um projeto com delineamento transversal, não sendo possível avaliar causa e efeito. Além disso, a avaliação do consumo alimentar é suscetível a erros de medidas. Pensando nisso, com o intuito de minimizar este viés, os R24H deste estudo foram aplicados por nutricionistas e/ou estudantes de nutrição devidamente treinados e a variação intrapessoal foi removida por métodos estatísticos, mas ainda assim é preciso reconhecer que, apesar das correções feitas no banco de dados, os valores obtidos podem conter imprecisões.

\section{CONCLUSÃO}

Os achados deste estudo demonstraram que maiores valores de IQC estão associados com maior adequação do consumo dos minerais avaliados, com exceção do selênio, demonstrando, assim, que a qualidade dos carboidratos presentes na dieta pode ser mais relevante do que a sua quantidade. Ademais, este achado pode ser visto como uma nova forma de abordagem terapêutica, onde o aumento do consumo de grãos integrais e fibras e a diminuição do consumo de açúcar resultarão na maior adequação de consumo de alguns minerais, além de diminuir os riscos relativos ao excesso de açúcares e carboidratos simples na dieta.

Sugere-se que novos estudos sejam realizados, com delineamentos que possam verificar uma sequência temporal e em outras populações, com intuito de sustentar ou contrariar os achados deste estudo.

\section{REFERÊNCIAS}

1. Ludwig DS, Hu FB, Tappy L, Brand-Miller J. Dietary carbohydrates: role of quality and quantity in chronic disease. BMJ. 2018;361:k2340.

2. Sales CH, Fontanelli MM, Vieira DA, Marchioni DM, Fisberg RM. Inadequate dietary intake of minerals: prevalence and association with socio-demographic and lifestyle factors. Br J Nutr. 2017;117(2):267-77.

3. Fisberg RM, Marchioni DM, Castro MA, Verly E Jr, Araújo MC, Bezerra IN, et al. Inadequate nutrient intake among the Brazilian elderly: National Dietary Survey 2008-2009. Rev Saúde Pública. 2013;47(suppl. 1):222s-30s.

4. Araujo MC, Bezerra IN, Barbosa FS, Junger WL, Yokoo EM, Pereira RA, et al. Macronutrient consumption and inadequate micronutrientintakeinadults. Rev SaúdePública. 2013;47(suppl. 1):177s-89s. 
5. Harvard Health Publishing. The larger role of micronutrients: You may need more of them in your daily diet than you think. [Acesso em 27 set 2019]. Disponível em: https://www.health. harvard.edu/staying-healthy/the-larger-role-of-micronutrients.

6. Gibson SA. Dietary sugars intake and micronutrient adequacy: a systematic review of the evidence. Nutr Res Rev. 2007;20(2):121-31.

7. Rennie KL, Livingstone MB.Associations between dietary added sugar intake and micronutrient intake: a systematic review. Br J Nutr. 2007;97(5):832-41.

8. Louie JC, Buyken AE, Brand-Miller JC, Flood VM. The link between dietary glycemic index and nutrient adequacy. Am J Clin Nutr. 2012;95(3):694-702.

9. Louie JC, Markovic TP, Ross GP, Foote D, Brand-Miller JC. Higher glycemic load diet is associated with poorer nutrient intake in women with gestational diabetes mellitus. Nutr Res. 2013;33(4):259-65.

10. Augustin LS, Kendall CW, Jenkins DJ, Willett WC, Astrup A, Barclay AW, et al. Glycemic index, glycemic load and glycemic response: an International Scientific Consensus Summit from the International Carbohydrate Quality Consortium (ICQC). Nutr Metab Cardiovasc Dis. 2015;25(9):795-815.

11. Zazpe I, Sanchez-Tainta A, Santiago S, de la Fuente-Arrilaga C, Bes-Rastrollo M, Martinez JA, et al. Association between dietary carbohydrate intake quality and micronutrient intake adequacy in a Mediterranean cohort: the SUN (Seguimiento Universidad de Navarra) Project. Br J Nutr. 2014;111(11):2000-9.

12. World Health Organization. Obesity: preventing and managing the global epidemic. Report of a WHO consultation, Geneva. Geneva: WHO; 1998.
13. Instituto Brasileiro de Geografia e Estatística. Pesquisa de Orçamentos Familiares 2008-2009. Tabelas de composição nutricional dos alimentos consumidos no Brasil. Rio de Janeiro: IBGE; 2011.

14. Haubrock J, Nothlings U, Volatier JL, Dekkers A, Ocke M, Harttig $\mathrm{U}$, et al. Estimating usual food intake distributions by using the Multiple Source Method in the EPIC-Potsdam Calibration Study. J Nutr. 2011;141(5):914-20.

15. Institute of Medicine. DRIDietary reference intakes: applications in dietary assessment. Washington: National Academies Press; 2000.

16. Goletzke J, Buyken AE, Louie JC, Moses RG, Brand-Miller JC. Dietary micronutrient intake during pregnancy is a function of carbohydrate quality. Am J Clin Nutr. 2015;102(3):626-32.

17. Sanchez-Villegas A, Zazpe I, Santiago S, Perez-Cornago A, Martinez-Gonzalez MA, Lahortiga-Ramos F. Added sugars and sugar-sweetened beverage consumption, dietary carbohydrate index and depression risk in the Seguimiento Universidad de Navarra (SUN) Project. Br J Nutr. 2018;119(2):211-21.

18. Kim DY, Kim SY, Lim H. Association between dietary carbohydrate quality and the prevalence of obesity and hypertension. J Hum Nutr Diet. 2018;31(5):587-96.

19. Zazpe I, Santiago S, Gea A, Ruiz-Canela M, Carlos S, BesRastrollo M, et al. Association between a dietary carbohydrate index and cardiovascular disease in the SUN (Seguimiento Universidad de Navarra) Project. Nutr Metab Cardiovasc Dis. 2016;26(11):1048-56.

Local de realização do estudo: Grupo de Educação e Estudos Oncológicos (GEEON) da Faculdade de Medicina da Universidade Federal do Ceará, Fortaleza, CE, Brasil.

Conflito de interesse: Os autores declaram não haver.

Foi apresentado como trabalho de destaque no XXIII Congresso Brasileiro de Nutrição Parenteral e Enteral, entre 20 a 23 de outubro de 2019, em Foz do Iguaçu-PR. 